\title{
Vitamin D supplementation in cutaneous malignant melanoma outcome (ViDMe): a randomized controlled trial
}

\author{
J. De Smedt ${ }^{1,2^{*}}$, S. Van Kelst ${ }^{1,2}$, V. Boecxstaens ${ }^{3,4}$, M. Stas ${ }^{3,4}$, K. Bogaerts ${ }^{5,6}$, D. Vanderschueren ${ }^{7,8}$, C. Aura ${ }^{9,10}$, \\ K. Vandenberghe ${ }^{11}$, D. Lambrechts ${ }^{12,13}$, P. Wolter ${ }^{14}$, O. Bechter ${ }^{15,16}$, A. Nikkels $^{17}$, T. Strobbe $^{18}$, G. Emri $^{19}$, \\ V. Marasigan ${ }^{1,2}$ and M. Garmyn ${ }^{1,2}$
}

\begin{abstract}
Background: Previous studies have investigated the protective effect of vitamin D serum levels, at diagnosis and during the follow-up period after treatment, on melanoma outcome. In the present study we assess whether vitamin $D$ supplementation, in the follow-up period after diagnosis and surgical resection of the primary tumor, has a protective effect on relapse of cutaneous malignant melanoma and whether this protective effect correlates with vitamin D levels in serum and Vitamin D Receptor immunoreactivity in the primary tumor.

Methods/design: This study is a multicenter randomized double blind placebo- controlled phase III trial. Patients between the age of 18 and 80 years diagnosed and treated surgically for a melanoma stage IB-III are eligible for randomization in a 1:1 ratio to active treatment or placebo. The study drug is taken each month and consists of either 100,000 International Unit cholecalciferol or arachidis oleum raffinatum used as a placebo. The primary endpoint is relapse free survival. The secondary endpoints are 25 hydroxyvitamin D3 serum levels at diagnosis and at 6 month intervals, melanoma subtype, melanoma site and stage of melanoma at diagnosis according to the 2009 American Joint Committee on Cancer melanoma staging and classification. At randomization a bloodsample is taken for DNA analysis. The study is approved by the local Ethics Committees.

Discussion: If we can confirm our hypothesis that vitamin D supplementation after removal of the tumor has a protective effect on relapse of cutaneous malignant melanoma we may reduce the burden of CMM at several levels. Patients, diagnosed with melanoma may have a better clinical outcome and improved quality of life. There will be a decrease in health care costs related to treatment of metastatic disease and there will be a decrease in loss of professional years, which will markedly reduce the economic burden of the disease.
\end{abstract}

Trial registration: Clinical Trial.gov, NCT01748448, 05/12/2012

Keywords: Melanoma, Vitamin D, Randomized controlled trial, Safety, Secondary prevention

\section{Background}

Cutaneous malignant melanoma: risk factors for development and progression, current knowledge

Cutaneous malignant melanoma (CMM) is the 7 th most frequent tumor in males and 4th most frequent tumor in females in Belgium [1]. It only accounts for $4 \%$ of all

\footnotetext{
* Correspondence: julie.desmedt@uzleuven.be

${ }^{1}$ Laboratory of Dermatology, Department of oncology, KU Leuven, 3000 Leuven, Belgium

2Department of Dermatology, University Hospitals Leuven, 3000 Leuven, Belgium

Full list of author information is available at the end of the article
}

malignant tumors of the skin, but it is responsible for $80 \%$ of the skin cancer related deaths $[2,3]$.

In 2013 the Belgian CMM incidence was 19.9 cases per 100,000 men (Crude Rate) and 18.5 cases per 100,000 women (Crude Rate) [4]. During the last decade's incidence rates have been rising in many countries within white populations and it is expected that the incidence will further increase the coming years $[5,6]$. CMM arises from a stepwise transformation of melanocytes in the skin, with subsequent superficial and deep invasion ultimately forming metastasis [3]. Several risk factors have been 
linked to the development of CMM. The strongest risk factors for melanoma are a family history of melanoma, multiple benign or atypical naevi and a previous melanoma. Immunosuppression, low phototype and exposure to ultraviolet light are additional risk factors [7]. The most important clinicopathological subtypes of melanoma are superficial spreading malignant melanoma, nodular malignant melanoma, lentigo maligna melanoma and acrolentiginous malignant melanoma. Diagnostic signs are progressive asymmetrical enlargement of a pigment lesion which also demonstrates irregularity in colour and irregularity in shape. Different risk factors are associated with different subtypes of CMM and different locations of malignant melanoma [8]. Different subtypes of malignant melanomas show also a significant genetic heterogeneity. The most important predictor for melanoma relapse and melanoma specific survival is Breslow thickness of the primary tumor at diagnosis [9]. This is a histological characteristic of the tumor and refers to the thickness of the tumor, measured as the distance between the granular layer of the epidermis and the deepest tumor cell in millimeters. Additional independent prognostic factors for survival are primary tumor ulceration and primary tumor mitotic rate according to the 2009 American Joint Committee on Cancer (AJCC) melanoma classification. Other known predictive prognostic factors are presence and distribution of tumor infiltrating lymphocytes, tumor site, sex and social deprivation $[9,10]$. The overall survival of CMM is better for earlier stages of the disease, however relapse poses a significant threat even for patients with limited disease. For this patient population we need an adjuvant treatment strategy which is cost/efficient, feasible and safe. Vitamin D supplementation is such a potential adjuvant treatment strategy, since vitamin D may protect against tumor progression through its pleiotropic anticancer effects via binding to its Vitamin D Receptor (VDR) [11].

\section{Vitamin D status: current knowledge}

Vitamin D, a fat-soluble seco-steroid, has two sources. The main source, vitamin D3 (cholecalciferol), is the skin. The other source, vitamin D2 (ergocalciferol) and D3, is exogenous and is ingested via dietary intake or supplements. The endogenous pathway is the most important source of vitamin D for humans. 7dehydrocholesterol (7-DHC), a provitamin in the epidermis, is photoactivated by ultraviolet B (UVB) radiation into provitamin D3. A series of modifications in the skin, liver and kidneys metabolizes vitamin D2 and vitamin D3 (dietary and endogenous) into the active substance $1 \alpha, 25$ dihydroxyvitamin D3 (calcitriol, 1,25(OH)2D3). $1,25(\mathrm{OH}) 2 \mathrm{D} 3$ and retinoid $\mathrm{X}$ receptor form a heterodimer and have an effect on gene expression by binding to the vitamin D receptor [11]. This is illustrated in Fig. 1.
The active metabolite of vitamin $\mathrm{D}, 1,25(\mathrm{OH}) 2 \mathrm{D} 3$, demonstrates relevant anti-cancer effects on melanoma cells. It has antiproliferative, differentiating and proapoptotic effects, but also inhibitory effects on tumour invasion and metastasis $[12,13]$. These pleiotropic anticancer effects are mediated by the VDR. Serum concentration of 25 hydroxy vitamin D3 $(25(\mathrm{OH}) \mathrm{D} 3)$ is the best indicator of the vitamin $\mathrm{D}$ status, which is determined by UVB induced production of vitamin D in the skin, dietary intake and vitamin $\mathrm{D}$ supplementation [14]. In addition vitamin D status may also be influenced by genetic variants of certain proteins involved in the vitamin D pathway [15]. A serum concentration of $25(\mathrm{OH}) \mathrm{D} 3$ below $10 \mathrm{ng} / \mathrm{ml}(<25 \mathrm{nmol} / \mathrm{L})$ is considered as vitamin $\mathrm{D}$ deficient, and a serum concentration below $20 \mathrm{ng} / \mathrm{ml}(<50 \mathrm{nmol} / \mathrm{L})$ as vitamin $\mathrm{D}$ insufficient. The optimal level for health is believed to be reached at $30 \mathrm{ng} / \mathrm{ml}(75 \mathrm{nmol} / \mathrm{L})$. Toxic levels are in the range of $100-150 \mathrm{ng} / \mathrm{ml}$ (or $250-375 \mathrm{nmol} / \mathrm{L}$ ). Vitamin D supplementation in the form of vitamin D3 can raise $25(\mathrm{OH}) \mathrm{D} 3$ concentrations and is safe when given in a single monthly dose of 100,000 IU during a year and given in an oral loading dose of 500,000 IU followed by an oral dose of 50,000 IU monthly for 2 years $[16,17]$. Current recommendation for additional vitamin D intake via supplements is $1000 \mathrm{IU} / \mathrm{d}$. Norman and Bouillon showed a good safety profile for this amount in more than 50,000 subjects over several years [18]. $25(\mathrm{OH}) \mathrm{D} 3$ concentrations reach a plateau after 3 months, when vitamin D3 supplementation is given monthly in a dose of $1000 \mathrm{IU} / \mathrm{d}$ [19]. There is an established proof of concept that vitamin D has an anticancer effect on CMM.

Already 30 years ago, in vitro studies demonstrated an anti-melanoma activity of $1,25(\mathrm{OH}) 2 \mathrm{D} 3$ with effects on cellular growth, differentiation, apoptosis, malignant cell invasion and metastasis [20]. These processes are possibly being mediated through the expression of VDR in malignant melanoma cells and primary melanoma tissue [21]. In vivo vitamin D has also shown to suppress growth of human malignant melanoma derived xenografts in immunosuppressed mice [22].

Previous studies have investigated the effect of vitamin D serum levels on CMM outcome, at diagnosis and/or during the follow up period after treatment [9, 23]. However conflicting results were obtained from these studies. The study of Newton Bishop et al. indicated that high vitamin D levels at diagnosis may have a protective effect on malignant melanoma outcome [9]. The study of Saiag et al. showed that the variability of $25(\mathrm{OH}) \mathrm{D} 3$ during follow-up of melanoma patients, but not the level at diagnosis per se was an independent prognostic marker [23]. A recent study showed that the associations of lower levels of vitamin 


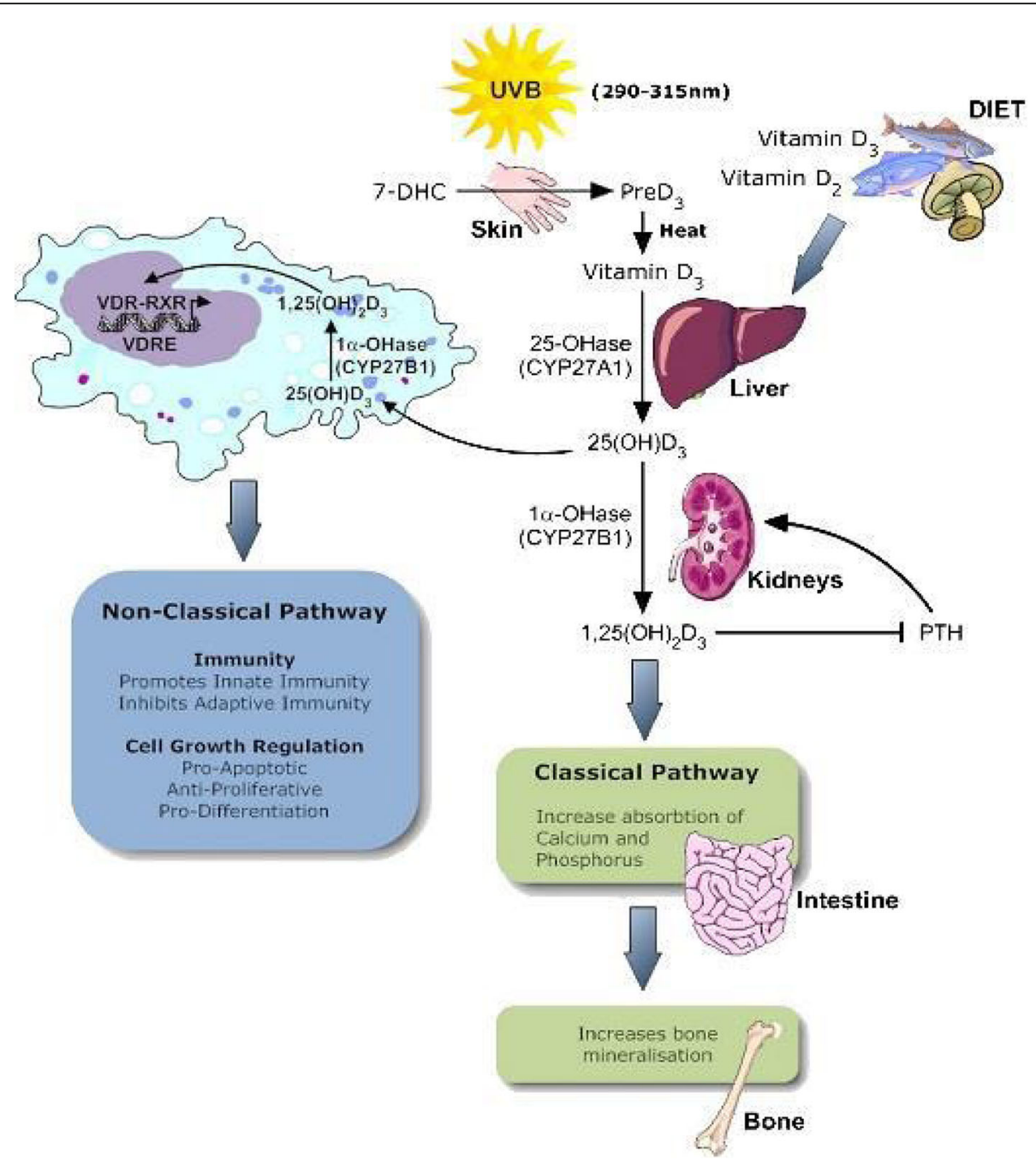

Fig. 1. Physiology of Vitamin D, reprinted from Melanoma and vitamin D, Molecular Oncology 5, Field S., Newton-Bishop JA., 2011; $197-214$ [11]. Copyright 2016, with permission from Elsevier

D with poorer overall survival, melanoma-specific survival and disease-free survival were independent from the association between lower vitamin $\mathrm{D}$ and higher C-reactive protein [24].To further elucidate a possible protective effect of vitamin $\mathrm{D}$ on melanoma outcome we initiated a multicenter randomized double blind placebo-controlled phase III trial to assess the effect of vitamin D supplementation on CMM relapse in the follow-up period after diagnosis and surgery of the primary tumor.

\section{Primary objectives}

The primary endpoint for this study is relapse-free survival. We will test the hypothesis that vitamin D supplementation after removal of the primary tumor has a protective effect on CMM relapse. We will also assess the immunohistochemical expression of VDR in the primary tumor and its possible correlation with relapse.

\section{Secondary objectives}

Vitamin D levels at diagnosis will be correlated with melanoma site, subtype and stage at diagnosis. We will monitor increases in vitamin D levels after supplementation. This will allow us to assess whether every patient is characterized by the same increase in vitamin $\mathrm{D}$ or whether serum levels following intake depend on the genetic variability in the vitamin D pathway and whether this genetic variability correlates with stage at diagnosis.

Furthermore, we will investigate whether VDR immunoreactivity correlates with stage at diagnosis.

\section{Methods and design}

\section{Study design}

This is a double blind placebo-controlled phase III multicenter study conducted at the University Hospitals Leuven, the University Hospitals of Antwerp, the Centre Hospitalier Universitaire de Liège in Belgium and at the 
Medical and Health Science Center at the University of Debrecen in Hungary.

\section{Overview of study flow}

There is a random assignment of patients in a 1:1 ratio. The treatment group receives a monthly oral dose of 100,000 IU of vitamin D (4 ml of D-Cure $\left.{ }^{\mathrm{ma}}\right)$ while the control group receives a monthly oral dose of placebo (4 $\mathrm{ml}$ of arachidis oleum). To overcome seasonal influences on baseline characteristics, a block randomization method stratifies on center and the time of diagnosis. Per center there are 3 strata: patients diagnosed within 6 months, between 6 and 12 months before start of the study and newly diagnosed patients (Fig. 2).

\section{Participants}

The aim is to enroll approximately 500 patients between the age of 18 and 80 years old. Eligible participants are patients with histologically proven malignant melanoma at high risk of recurrence namely in stage IB-III (stage IA patients will not be included in the study because they only have a very small chance of relapsing). Inclusion in the study is only allowed after a complete resection of the melanoma has occurred. Participants are included up to 1 year after diagnosis and after signing an informed consent, which had been approved by the local ethics committee. For detailed inclusion and exclusion criteria see Appendix 1.

\section{Materials}

Participants randomized to the active treatment arm, will receive 1 oral syringe filled with 4 ampoules of D-cure ${ }^{\text {rax }}$ (Laboratories SMB NV, Brussels, Belgium), corresponding to 100,000 IU cholecalciferol, on a monthly basis. Content of 1 such ampoule is cholecalciferol 25,000 IU, DL- $\alpha$ tocopherol acetate, sorbitol oleate, orange peel oil and arachis oil ad $1 \mathrm{ml}$. Participants randomized to the placebo arm, will receive 1 oral syringe containing $4 \mathrm{ml}$ arachis oil (manufactured by Fagron, Waregem, Belgium).

\section{Study drug administration and randomization}

An independent site pharmacist prepares the medication. A treatment and corresponding medication is assigned to the patient during randomization. Medication is taken monthly consisting of either a dose of 100,000 IU (4 ml of D-cure ${ }^{\mathrm{rm}}$ suspension in arachis oil) or $4 \mathrm{ml}$ of placebo (arachis oil). The first intake of the drug is given immediately at randomization in the hospital. Subsequent doses are taken monthly at home and are registered in a patient diary. Oral syringes are numbered according to the randomization schedule. At each visit, 3 (if a control is foreseen every 3 months in the university setting) or 6 syringes (if a control is only foreseen every 6 months in the university setting and meanwhile by a peripheral dermatologist) are given to the patient. The date when the vitamin D/placebo is taken and the number of syringes used are registered. Empty syringes are destroyed.

\section{Screening and randomization of patients}

From Q4 2012 to Q4 2016 eligible stage IB-III melanoma patients will be enrolled in our study. After staging, surgical treatment and signing an informed consent form, patients are screened and all in- and exclusion criteria (see Appendix 1) verified. When deemed to be eligible, the patient is randomized by the Leuven Coordinating Centre (LCC, Leuven, Belgium) using an Interactive

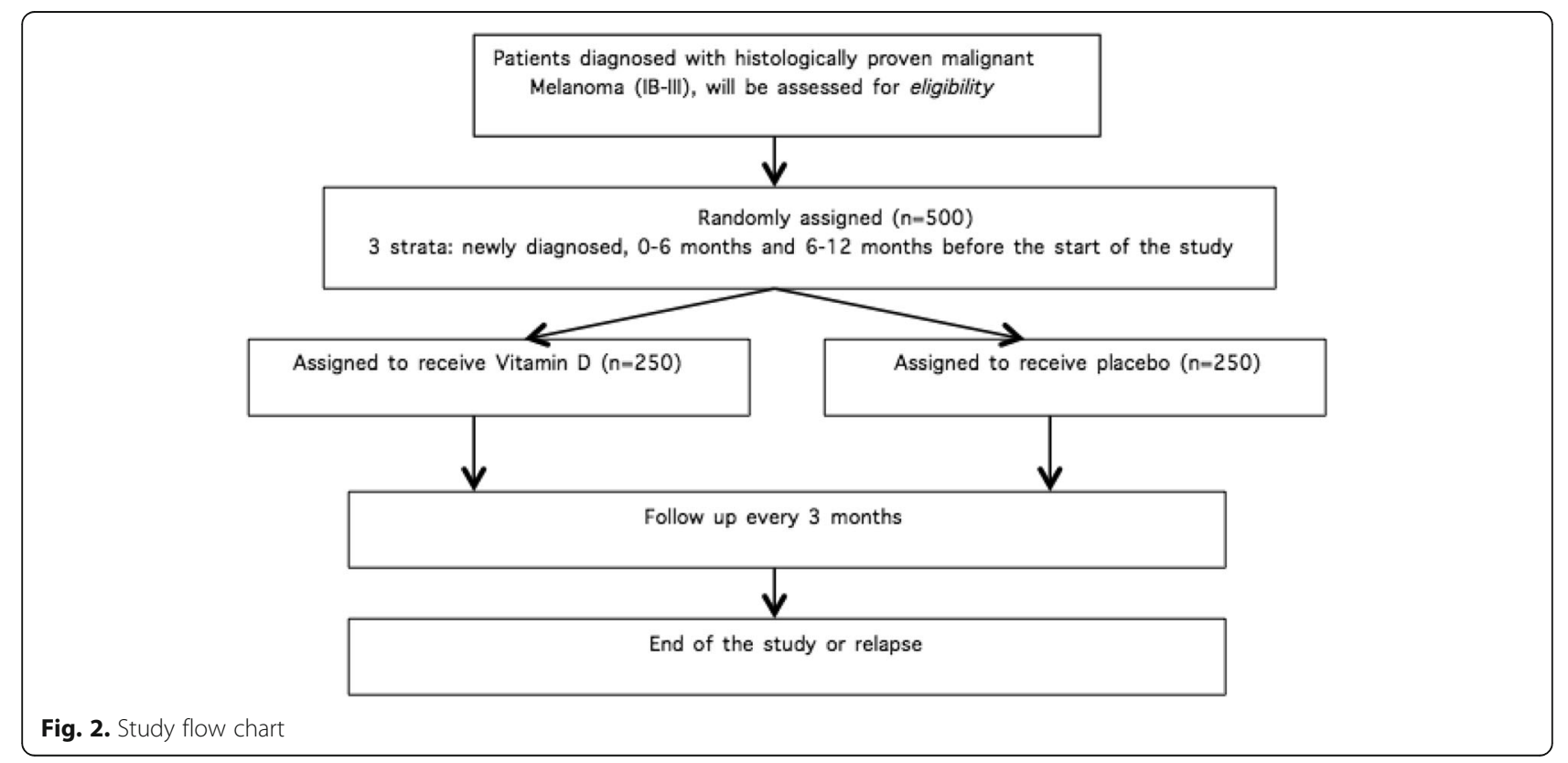


Voice Response System (IVRS) on an equal basis (1:1) to either vitamin $\mathrm{D}$ or placebo group, stratified on time of diagnosis (newly diagnosed, 0-6 months or 6-12 months before start of the study) and centre. All study staff members, trial nurses, dermatologists and patients are blinded to the treatment allocation during the whole study duration. The data management center keeps the randomization schedule confidential. The study complies with the Helsinki declaration of medical research and is approved by the hospital medical ethics committees of the 4 participating centres.

\section{Study conduct}

At randomization a questionnaire is completed for every participant with questions on melanoma risk factors, including the amount and type of sun exposure and a full skin examination for the assessment of phototype, naevus phenotype and actinic damage is completed. Body Mass Index (BMI) is estimated after measuring the patient's height and weight.

To monitor the effects of high dose vitamin D supplementation, diagnostic tests and assays are performed at regular time intervals. Serum 25(OH)D3 levels are measured at randomization and every 6 months subsequently. Every 3 months and at the end of the study serum calcium and serum phosphate are measured. Renal and liver function tests are evaluated at randomization and at the end of the study. At the end of the study, a 24-h urinary calcium excretion test is done. For detailed information about schedule assessments see Table 1. At randomization, the study team will give the patient a diary in which to record their use of drugs and side effects which are reviewed by the clinician at follow-ups. Dose adherence of the patients is controlled by recording the number of empty syringes which the patients need to bring back. Adherence to the study drug is improved by seeing the patients at regular intervals ( 3 monthly) either by the general practitioner, private dermatologist or at a university hospital setting. Patients are followed until relapse or end of study.

\section{Safety}

Baseline laboratory tests are performed at screening of the patient and safety laboratory every 3 months of follow-up. The parameters that are analyzed can be found in Table 1. Laboratory parameters are only reported as abnormal if the investigator assesses them as 'clinically significant' and/or when they result in precautionary safety measures. The following laboratory values will be reported as adverse events (AEs):

- Hypercalcemia: defined as 2 independent measurements above the reference value as defined by the laboratory where the sample is determined (first measurement at the investigational site and second measurement at general practitioner/dermatologist)

- Hypervitaminosis D: defined as serum 25(OH)2D3 level $>80 \mathrm{ng} / \mathrm{ml}$

- Hypovitaminosis D: Deficient: serum 25(OH)2D3 $<10 \mathrm{ng} / \mathrm{ml}$ Insufficient: serum $25(\mathrm{OH}) 2 \mathrm{D} 3 \geq 10 \mathrm{ng} / \mathrm{ml}$ and $<20 \mathrm{ng} / \mathrm{ml}$

- Hypophosphatemia: defined as phosphate level $<1.5 \mathrm{mg} / \mathrm{dl}$

- Hyperphosphatemia: defined as phosphate level $>6 \mathrm{mg} / \mathrm{dl}$

Table 1 Schedule of assessments

\begin{tabular}{|c|c|c|c|c|c|}
\hline & Screening & Randomization & $\begin{array}{l}3 \text { monthly } \\
\text { FU }\end{array}$ & $\begin{array}{l}6 \text { monthly } \\
\text { FU }\end{array}$ & End of study \\
\hline $25(\mathrm{OH}) \mathrm{D} 3$ serum levels & & $x$ & $\begin{array}{l}\text { X } \\
\text { (when 25(OH)D3 levels }>80 \mathrm{ng} / \mathrm{ml} \text { and } \\
\text { study drug temporarily interrupted) }\end{array}$ & $x$ & \\
\hline Blood sample for DNA analysis & & $x$ & & & \\
\hline Hemoglobin, Hematocrit & $x$ & & & & $x$ \\
\hline WBC count and differentiation & $x$ & & & $x$ & $x$ \\
\hline RBC count & $x$ & & & & $x$ \\
\hline Platelet count & $x$ & & & & $x$ \\
\hline Renal function (creatinine) & $x$ & & & & $x$ \\
\hline Liver function (AF, ALT, gamma-GT, LDH) & $x$ & & & & $x$ \\
\hline Serum calcium & $x$ & & $x$ & & $x$ \\
\hline Serum phosphate & $x$ & & $x$ & & $x$ \\
\hline Albumin & $x$ & & & & $x$ \\
\hline $24 \mathrm{~h}$ urine calcium & & & & & $x$ \\
\hline
\end{tabular}


Immunosuppressive effects are evaluated by reporting the rate of infection every 3 months and by measuring the total White Blood Cell (WBC) count and differential every 6 months. For detailed information see Appendix 2. All AEs are documented by study investigators. An $\mathrm{AE}$ is defined as any untoward medical occurrence in a patient administered with an investigational medicinal product and which does not necessarily have a causal relationship with this treatment. Severity of AEs is assessed (mild, moderate, severe or unknown). A Serious Adverse Event (SAE) is defined as any untoward medical occurrence that at any dose results in death, is life-threatening, requires inpatient hospitalization or prolongation of existing hospitalization, results in persistent or significant disability/incapacity, congenital anomal/birth defect or is medically significant. SAEs are documented within $24 \mathrm{~h}$ in the SAE module provided in the electronic Case Report Form (eCRF) and reported to the LCC. All SAEs are monitored and followed up until the outcome is known. The Data and Safety Monitoring Board (DSMB) reviews all safety parameters and AEs. This board is composed of a dermatologist, an oncologist and biostatistician, who are all not involved in the study and are unblinded. At regular intervals, an unblinded interim analysis is performed to assess the difference between the intervention arms and to exclude an increase in relapse rate in the vitamin D supplemented arm.

\section{VDR expression in primary melanoma}

The expression of the VDR is immunohistochemically assessed in the primary tumor of each melanoma patient that is enrolled in the study. This is done on formalinfixed paraffin embedded (FFPE) tissue that is collected at diagnosis and is stored according to the standard procedures of the department of pathology (Professor J. van den Oord). FFPE slides from every melanoma are stained on an autostainer (operational) using the Envision technique and a commercial monoclonal anti-VDR antibody. The levels of VDR expression and their localization in the different phases are semi-quantitatively assessed by an experienced pathologist (Dr. C. aura).

\section{Determination of $25-(\mathrm{OH}) \mathrm{D}$ serum levels}

Serum 25(OH)D3 levels are determined at randomization and every 6 months, using a commercially available radioimmunoassay (RIA) kit: Diasorin, Stillwater, MN (USA) as described [16]. This RIA is well accepted as a reference protocol and commonly used to determine 25(OH)D3 levels in the field. The laboratory of "Experimental medicine and Endocrinology" (Professor D. Vanderschueren) has extensive experience in measuring 25(OH)D3 levels and will therefore perform all these measurements [16]. The intra- and inter-assay coefficients of variation for
$25(\mathrm{OH}) \mathrm{D}$ are $11 \%$ and $8 \%$ respectively. The detection of the RIA kit is $5.0 \mathrm{nmol} / \mathrm{L} 25(\mathrm{OH}) \mathrm{D}$. In addition, serum $25(\mathrm{OH}) \mathrm{D} 3$ levels will also be determined with an in house liquid chromatography/tandem mass spectrophotometry method which is currently been developed and validated in the diagnostic laboratory of the Leuven University hospital.

\section{DNA analysis}

A bloodsample for DNA analysis is taken at randomization. Genetic variants affecting vitamin D levels are genotyped by using iPLEX technology. Multiple single nucleotide polymorphisms (SNPs) have been reported to affect vitamin D signaling. We analyse 4 common SNPs (rs12785878, rs10741657, rs6013897 and rs7041), because they have the most important effect on 25(OH)D3 levels. Fifteen candidate SNPs, located in genes in the vitamin D pathway and previously reported to affect vitamin D signaling, are selected as well. Those 15 SNPs may independently determine 25(OH)D3 levels.

\section{Participant follow-up}

On a 3 monthly basis, there is a follow-up of all patients included in the study. Consisting of physical examination, including full skin assessment and palpation of regional lymph nodes and blood sample tests (see Table 1). At intervals of 6 months (or upon clinical indication), an ultrasound of the lymph nodes will be performed. Only when new symptoms arise or physical findings are suggestive for progression, a Positron Emission Tomography-Computed Tomography (PET-CT) or Magnetic Resonance Imaging (MRI) is performed to exclude distant metastasis. Every 12 months, an ultrasound of the abdomen and x-ray of the chest (or CT thorax/abdomen if clinically indicated) are performed.

\section{Blinding}

Both patients and investigators are blinded to study medication and vitamin D levels.

\section{Study duration}

Patients enrolled in the study will be followed up throughout the entire study duration, with a maximum of 3,5 years follow-up. Participation of a patient may be terminated earlier due to relapse/progression or due to a reasonable cause, such as the investigator's medical decision. At any time, the patient has the right to withdraw consent without a negative impact on her/his medical treatment. The planned enrolment rate is 500 melanoma patients during a recruitment phase of 4 years maximum. The first patient was recruited in fall 2012 and the last patient will be recruited in fall 2016. The study will stop 6 months after the last patient is randomized. The study may terminate earlier due to interruption of the DSMB, the sponsor, 
competent authorities/ethics committees or if the work is not compliant with Good Clinical Practice.

\section{Sample size justification}

Assuming similar times to relapse as retrospectively observed at the University hospital Leuven, 3 years of recruitment and a total study duration of 3.5 years, 500 patients will have $90 \%$ power to detect a hazard ratio of 0.40 in favour of the vitamin D supplemented arm by means of Cox proportional hazards model stratified for time since diagnosis (3 strata: $0,0-6$ or $6-12$ months ago). The hazard ratio of 0.40 is in correspondence with the intermediate effect size that was reported by Newton-Bishop et al. [9] and a potential increase in VD serum levels of $70 \mathrm{nmol} / \mathrm{L}$, which is $80 \%$ of the observed effect in a study with COPD patients [16]. It was anticipated that no patient will be lost for follow-up. Due to a slow recruitment, the recruitment period was extended by 1 year.

\section{Statistical methods}

The primary analysis is an intent-to-treat analysis of all randomized patients. Patients will be analysed according to which treatment group they were randomized, irrespective of which study drug or even if any study drug was received. A Cox proportional hazard model with stratification for time since diagnosis (3 strata: 0, 0-6 or 6-12 months ago) will be performed to investigate the difference between the two treatment groups for the primary endpoint, which is relapse-free survival. If the treatment effect is stratum dependent, then the strata will be analysed separately. A correction for baseline covariates like sex, age and the baseline vitamin $\mathrm{D}$ level will be performed as sensitivity analyses. A Cox proportional hazard model will also be used to investigate whether a potential difference in relapse-free survival between two treatment groups is different for VDR immunoreactivity and genetic variability in the vitamin $\mathrm{D}$ pathway. The hazard ratios and associated 95\%-confidence intervals $(\mathrm{CI})$ will be determined to assess the contributions of significant factors. All reported $p$-values will be two-sided, and a $p$-value less than 0.05 will be considered statistically significant. A linear mixed model will be used to identify the evolution of $25(\mathrm{OH}) \mathrm{D} 3$ levels in function of genetic variability in the vitamin $\mathrm{D}$ pathway. In order to examine the correlation between VDR immunoreactivity and stage at diagnosis, a KruskalWallis test will be used. For newly diagnosed melanoma patients, an analysis of variance will be performed to correlate vitamin D levels at diagnosis, melanoma site and subtype. Full details of the statistical analyses will be described in a statistical analysis plan which will be finalized before database lock.

\section{Quality assurance}

All efforts are being made to reassure maximal quality control. The study is performed according to good manufacturing practices. Direct access to source data, with strict adherence to all confidentiality regulations, is available for monitoring purposes. In addition, a DSMB reviews all safety parameters and AEs.

\section{Discussion}

To the best of our knowledge, this is one of the first randomized, placebo-controlled phase III trial to examine the efficacy and safety of long-term and high-dose vitamin D supplementation in melanoma patients, with a maximum time of supplementation of 3,5 years [17]. Patients are randomized in a 1:1 ratio, either receiving 100,000 IU cholecalciferol or placebo. The patients' clinical status, including adverse events, is monitored at regular intervals. Blood tests and other technical investigations are also regularly conducted as explained previously (see Table 1). There is a rising interest in vitamin $\mathrm{D}$ with respect to its pleiotropic effects on chronic diseases and cancer. The objective of the project is to build further on the knowledge of these effects in the field of melanoma treatment. Keeping in mind, the compromised prognosis of patients with relapsing malignant melanoma and the health as well as economic burden associated with metastatic disease we consider vitamin D supplementation a considerably save, low cost and broadly acceptable adjuvant strategy worthwhile being tested. If we can confirm our hypothesis that vitamin D supplementation after removal of the tumor has a protective effect on relapse of CMM, we may markedly reduce the burden of CMM at several levels. Patients, diagnosed with melanoma may have a better clinical outcome, which not only means a decrease in loss of years but also a decrease in loss of professional years (decreased mortality in still professionally active young population). Patients diagnosed with melanoma will have an improved quality of life (less relapse, less complications due to treatment of regional metastatic and distant metastatic disease). Finally, there will be a decrease in health care costs related to treatment of regional and distant metastatic disease.

\section{Registration}

The study is registered with Clinical Trial.gov (NCT01748448). Eudract No: 2012-002125-30.

\section{Protocol}

A full copy of the protocol of the current ViDMe study can be requested from the principal investigator Prof. Dr. Marjan Garmyn, email: marjan.garmyn@kuleuven.be. 


\section{Appendix 1}

Inclusion and exclusion criteria for the ViDMe study

\section{Inclusion criteria}

1. Older than 18 years and younger than 80 years of age.

2. Histologically proven malignant melanoma, stage IB to III. Not participating in another clinical trial.

3. The only treatment for melanoma is surgical treatment.

4. Complete resection of melanoma.

5. Single primary invasive cutaneous melanoma (inclusion within 1 year after diagnosis).

6. Signed ethical committee approved informed consent.

7. Serum phosphate and calcium at the entry of the study within normal range of the laboratory reference.

\section{Exclusion criteria}

1. Pregnant/lactating women or planning on becoming pregnant during the study.

2. Known hypersensitivity to vitamin D or its components.

3. Pre-existing renal stone disease or chronic renal disease.

4. Impaired renal function with an eGRF $<30 \mathrm{~mL} / \mathrm{min} /$ $1.73 \mathrm{~m} 2$ or renal dialysis.

5. Liver failure or chronic liver disease with liver enzyme $>2$ fold the upper limit of normal.

6. History of parathyroid disease or granulomatous disease (tuberculosis or sarcoidosis).

7. History of malabsorption syndrome or any medical condition that might interfere with Vitamin D absorption.

8. History of other malignancy within the last 5 years except for carcinoma in situ of the cervix or basal cell carcinoma or squamous cell carcinoma of the skin or in situ malignant melanoma.

9. Chronic alcohol abuse.

10.Medical or logistic problems likely to preclude completion of the study

\section{Appendix 2}

Safety procedures in the event of abnormal assay results

\section{End of study}

- Clinical significant hypercalcemia: defined as 2 independent measurements above the reference value

- Hypovitaminosis D (serum 25(OH)2D3 < $10 \mathrm{ng} / \mathrm{ml}$ )

- Pregnancy

- Disease progression

\section{Action taken}

- Hypervitaminosis D: Defined as 25(OH)2D3 > 80 $\mathrm{ng} / \mathrm{ml}$. Discontinue study drug and restart monthly treatment with study drug when $25(\mathrm{OH}) 2 \mathrm{D} 3$ falls below $50 \mathrm{ng} / \mathrm{ml}$ based on a 3 monthly monitoring.

- Hypovitaminosis D: serum 25(OH)2D3 > $10 \mathrm{ng} / \mathrm{ml}$ and $<20 \mathrm{ng} / \mathrm{ml}$ : supplementation of extra vitamin D (D-cure): 25,000 IU/month

- Hypophosphatemia: < $1.5 \mathrm{mg} / \mathrm{dl}$ : further investigation

- Hyperphosphatemia: > $6 \mathrm{mg} / \mathrm{dl}$ : further investigation

\section{Concomittant medications/treatments}

Concomitant use of vitamin D or multi-vitamin supplements, concomitant use of calcium supplements and medications which we know interact with study medication are registered.

Other medications then listed below, will not be recorded:

- Digoxin

- Lithium

- Thiazide diuretics

- Anticonvulsants

- Corticosteroids (Chronic oral use is an exclusion criteria, topical use is permitted)

- H2 receptor antagonists

\section{Abbreviations}

1,25(OH)2D3: 1a,25 dihydroxyvitamin D3; 25(OH)D3: 25 hydroxyvitamin D3; 7-DHC: 7-dehydrocholesterol; AE: Adverse Event; AF: Alkaline phosphatase; AJCC: American Joint Committee on Cancer; ALT: Alanine Aminotransferase; BMI: Body Mass Index; CMM: Cutaneous Malignant Melanoma; COPD:

Chronic Obstructive Pulmonary Disease; CTA: Clinical Trial Assistant; DSMB: Data and Safety Monitoring Board; eCRF: electronic Case Report Form; FFPE: Formalin-Fixed Paraffin Embedded; Gamma-GT: GammaGlutamyl Transferase; IU: International Unit; IVRS: Interactive Voice Response System; LCC: Leuven Coordinating Centre; LDH: Lactaat Dehydrogenase; MRI: Magnetic Resonance Imaging; PET-CT: Positron Emission TomographyComputed Tomography; RBC: Red Blood Cell; RIA: Radioimmunoassay; SAE: Serious Adverse Event; UVB: Ultraviolet B; VDR: Vitamin D Receptor; WBC: White Blood Cell Count

\section{Acknowledgements}

The authors gratefully acknowledge the contributions of all residents of the Department of Dermatology of the Catholic University of Leuven, the University Hospitals of Antwerp, the Centre Hospitalier Universitaire de Liège and the Medical and Health Science Center at the University of Debrecen. The authors also acknowledge for the supporting help of Karin Broos (LCC), Tine Vanhoutvin (CTA at the Department of Dermatology of the Catholic University of Leuven), Mara Airo (CTA at the Department of Dermatology of the Centre Hospitalier Universitaire de Liège) and Corinne Chapelier (CTA at the Department of Dermatology of the Centre Hospitalier Universitaire de Liège).

\section{Funding}

IWT-TBM project 110,688 "Vitamin D supplementation in cutaneous malignant melanoma outcome".

Availability of data and materials Not applicable. 


\section{Authors' contributions}

JDS participated in the recruitment and follow up of patients and assisted in drafting the final manuscript. SVK participated in the recruitment and follow up of patients and assisted in the coordination of the study. KB, DV, CA, KV, DL participated in the design of the study: KB in the statistical analysis, DV in the 25(OH)D3 serum level detection and safety, CA in the VDR receptor expression assessment, $\mathrm{KV}$ in the management of the data, and $\mathrm{DL}$ in the DNA analysis. BV, MS, PW, OB, AN, TS and ER participated in the recruitment of the patients.VM participated in the design and coordination of the study, and in the recruitment and follow up of the patients. MG is responsible for the design, coordination and final analysis of the study and acquired the funding. All authors read an approved the final manuscript.

\section{Authors' information}

JDS is MD and research assistant at the department of Dermatology of the University Hospitals Leuven. SVK is a clinical trial assistant and technician in the laboratory of Dermatology of KU Leuven. BV and SM are surgical oncologists at the University Hospitals Leuven. KB is a Project Manager external consultancy at KU Leuven. DV is an endocrinologist at the University Hospitals Leuven with special expertise in metabolic bone diseases. CA is pathologist and post-doc at the Laboratory of Transitional Cell \& Tissue Pathology, KULeuven, and is supported by a EU-FP7 Marie Curie IAPP (SYS-MEL) grant. KV is Project Manager at the LCC of the Department of cardiovascular sciences of the University Hospitals Leuven. DL received a Master in Engineering and PhD in Medical Sciences at the KU Leuven. Currently he is Full Professor at the Laboratory for Translational Genetics at KU Leuven, and Director of the Vesalius Research Center, VIB, Belgium. PW was an oncologist at the University Hospitals Leuven, currently is an oncologist at the CHR Verviers East Belgium in Verviers. OB is an oncologist at the University Hospitals Leuven. AN is dermatologist and head of the department of Dermatology of the Centre Hospitalier Universitaire de Liège. TS is a dermatologist specialized in dermatosurgery and melanoma at the University Hospitals Antwerp. GE is a dermatologist and oncologist, head of the Melanoma Unit of the department of Dermatology of the University of Debrecen. VM is a MD and PhD student at the department of Dermatology of KU Leuven. MG is dermatologist and head of the Department of Dermatology of the University Hospitals of Leuven.

\section{Ethics approval and consent to participate}

The study is approved by the UZ Leuven Medical Ethics committee. The committee's reference number: ML8638. A statement on ethics approval and consent can be requested from the principal investigator Prof. Dr. Marjan Garmyn, email: marjan.garmyn@kuleuven.be.

Before randomization all patients have signed an informed consent.

\section{Consent for publication}

Not applicable.

\section{Competing interests}

The authors declare that they have no competing interests.

\section{Publisher's Note}

Springer Nature remains neutral with regard to jurisdictional claims in published maps and institutional affiliations.

\footnotetext{
Author details

'Laboratory of Dermatology, Department of oncology, KU Leuven, 3000 Leuven, Belgium. ${ }^{2}$ Department of Dermatology, University Hospitals Leuven, 3000 Leuven, Belgium. ${ }^{3}$ Oncological and vascular access surgery, Department of surgical oncology, University Hospitals Leuven, 3000 Leuven, Belgium. ${ }^{4}$ Department of oncology, KU Leuven, 3000 Leuven, Belgium. ${ }^{5} \mathrm{KU}$ Leuven, Faculty of Medicine, I-BioStat, 3000 Leuven, Belgium. ${ }^{6}$ Hasselt University, I-BioStat, 3590 Diepenbeek, Belgium. ${ }^{7}$ Clinical and Experimental Endocrinology, Department of Clinical and Experimental Medicine, KU Leuven, 3000 Leuven, Belgium. ${ }^{8}$ Department of Endocrinology, University Hospitals Leuven, 3000 Leuven, Belgium. ${ }^{9}$ Translational Cell \& Tissue Research, Department of Imaging \& Pathology, KU Leuven, 3000 Leuven, Belgium. ${ }^{10}$ Department of Pathology, University Hospitals of Leuven, 3000 Leuven, Belgium. ${ }^{11}$ Department of Cardiovascular Sciences, KU Leuven, 3000 Leuven, Belgium. ${ }^{12}$ Laboratory for Translational Genetics, Department of Oncology, KU Leuven, 3000 Leuven, Belgium. ${ }^{13}$ Vesalius Research Center, VIB, 3000 Leuven, Belgium. ${ }^{14}$ Department of Hematology and Oncology, CHR
}

Verviers East Belgium, 4800 Verviers, Belgium. ${ }^{15}$ Laboratory of Experimental Oncology (LEO), Department of Oncology, KU Leuven, 3000 Leuven, Belgium. ${ }^{16}$ Department of General Medical Oncology, University Hospitals Leuven, Leuven Cancer Institute, 3000 Leuven, Belgium. ${ }^{17}$ Department of

Dermatology, CHU Sart Tilman, University of Liège, 4000 Liège, Belgium.

${ }^{18}$ Department of Dermatology, University Hospital Antwerp, 2650 Edegem, Belgium. ${ }^{19}$ Department of Dermatology, Faculty of Medicine, University of Debrecen, Debrecen 4012, Hungary.

Received: 15 July 2016 Accepted: 9 August 2017

Published online: 23 August 2017

\section{References}

1. Belgian Cancer Registry: De tien meest frequente tumoren per geslacht, België 2013. http://www.kankerregister.org/statistieken.

2. Jemal A, Siegel R, Ward E, Hao Y, Xu J, Murray T, Thun MJ. Cancer statistics, 2008. CA Cancer J Clin. 2008;58:71-96.

3. Miller AJ, Mihm MC Jr. Melanoma. N Engl J Med. 2006;355(1):51-65.

4. Doe J. Incidence fact sheet malignant melanoma. In: Belgian Cancer Registry incidence fact sheets. 2013. http://www.kankerregister.org/media/docs/ Incidencefactsheets/Incidence_Fact_Sheet_MalignantMelanoma_2013.pdf. Accessed 22 Sept 2015.

5. Marks R. Epidemiology of melanoma. Clin Exp Dermatol. 2000;25:459-63.

6. Garbe C, Leiter U. Melanoma epidemiology and trends. Clin Dermatol. 2009;27:3-9.

7. Bataille V, de Vries E. Melanoma part 1: epidemiology, risk factors, and prevention. BMJ. 2008; doi:10.1136/bmj.a2249.

8. Whiteman DC, Watt P, Purdie DM, Hughes MC, Hayward NK, Green AC. Melanocytic nevi, solar keratoses, and divergent pathways to cutaneous melanoma. J Natl Cancer Inst. 2003;95(11):806-12.

9. Newton-Bishop JA, Beswick S, Randerson-Moor J, Chang YM, Affleck P, Elliott F, Chan M, Leake S, Karpavicius B, Haynes S, Kukalizch K, Whitaker L, Jackson S, Gerry E, Nolan C, Bertram C, Marsden J, Elder DE, Barrett JH, Bishop DT. Serum 25-hydroxyvitamin D3 levels are associated with Breslow thickness at presentation and survival from melanoma. J Clin Onc. 2009;27(32):5439-44.

10. Balch CM, Gershenwald JE, Soong SJ, Thompson JF, Atkins MB, Byrd DR, Buzaid AC, Cochran AJ, Coit DG, Ding S, Eggermont AM, Flaherty KT, Gimotty PA, Kirkwood JM, McMasters KM, Mihm MC Jr, Morton DL, Ross Ml, Sober AJ, Sondak VK. Final version of 2009 AJCC melanoma staging and classification. J Clin Oncol. 2009;27(36):6199-206.

11. Field S, Newton-Bishop JA. Melanoma and vitamin D. Mol Oncol. 2011:5:197-214

12. Osborne JE, Hutchinson PE. Vitamin D and systemic cancer: is this relevant to malignant melanoma? Br J Dermatol. 2002;147:197-213.

13. Egan KM. Vitamin D and melanoma. Ann Epidemiol 2009; 19 (7): 455-461.

14. Sage RJ, Lim HW. Therapeutic hotline: recommendations on photoprotection and vitamin D. Dermatol Ther. 2010;23(1):82-5.

15. Wang TJ, Zhang F, Richards JB, Kestenbaum B, van Meurs JB, Berry D, Kiel DP, Streeten EA, Ohlsson C, Koller DL, Peltonen L, Cooper JD, O'Reilly PF, Houston DK, Glazer NL, Vandenput L, Peacock M, Shi J, Rivadeneira F, MI MC, Anneli P, de Boer $I H$, Mangino M, Kato B, Smyth DJ, Booth SL, Jacques PF, Burke GL, Goodarzi M, Cheung CL, Wolf M, Rice K, Goltzman D, Hidiroglou N, Ladouceur M, Wareham NJ, Hocking L, Hart D, Arden NK, Cooper C, Malik S, Fraser WD, Hartikainen AL, Zhai G, Macdonald HM, Forouhi NG, Loos RJ, Reid DM, Hakim A, Dennison E, Liu Y, Power C, Stevens HE, Jaana L, Vasan RS, Soranzo N, Bojunga J, Psaty BM, Lorentzon M, Foroud T, Harris TB, Hofman A, Jansson JO, Cauley JA, Uitterlinden AG, Gibson Q, Järvelin MR, Karasik D, Siscovick DS, Econs MJ, Kritchevsky SB, Florez JC, Todd JA, Dupuis J, Hyppönen E, Spector TD. Common genetic determinants of vitamin $D$ insufficiency: a genome-wide association study. Lancet. 2010;376(9736):180-8.

16. Lehouck A, Mathieu C, Carremans C, Baeke F, Verhaegen J, Van Eldere J, Decallone B, Bouilon R, Decramer M, Janssens W. High doses of vitamin D to reduce exacerbations in chronic obstructive pulmonary disease: a randomized trial. Ann Intern Med. 2012;156(2):105-14.

17. Saw RPM, Armstrong BK, Mason RS, Morton RL, Shannon KF, Spillane AJ, Stretch JR, Thompson JF. Adjuvant therapy with high dose vitamin D following primary treatment of melanoma at high risk of recurrence: a placebo controlled randomized phase II trial (ANZMTG 0.09 Mel-D). BMC Cancer. 2014;14:780. 
18. Norman AW, Bouillon R. Vitamin D nutritional policy needs a vision for the future. Exp Biol Med (Maywood). 2010;235(9):1034-45.

19. Heaney RP, Davies KM, Chen TC, Holick MF, Barger-Lux MJ. Human serum 25-hydroxycholecalciferol response to extended oral dosing with cholecalciferol. Am J Clin Nutr. 2003;77(1):204-10.

20. Colston K. Colston Mj, feldman D. 1,25-dihydroxyvitamin D3 and malignant melanoma: the presence of receptors and inhibition of cell growth in culture. Endocrinology. 1981;108(3):1083-6.

21. Caini S, Boniol M, Tosti G, Magi S, Medri M, Stanganelli I, Palli D, Assedi M, Del Marmol V, Gandini S. Vitamin D and melanoma and non-melanoma skin cancer risk and prognosis: a comprehensive review and metanalysis. Eur J Cancer. 2014;50(15):2649-58.

22. Eisman JA, Barkla DH, Tutton PJM. Suppression of in vivo growth of human cancer solid tumor Xenografts by 1,25-Dihydroxyvitamin D3. Cancer Res. 1987:47:21-5

23. Saiag P, Aegerter P, Vitoux D, Lebbé C, Wolkenstein P, Dupin N, Descamps V, Aractingi S, Funck-Brentano E, Autier P, Dragomir M, Boniol M. Prognostic value of 25-hydroxyvitamin D3 levels at diagnosis and during follow-up in melanoma patients. JNCI J Natl Canc Inst. 2015;107(12):djv264.

24. Fang S, Sui D, Wang Y, Liu H, Chiang Y-J, Ross Ml, Gershenwald JE, Cormier JN, Royal RE, Lucci A, Wargo J, Hu Ml, Gardner JM, Reveille JD, Bassett RL, Wei Q, Amos Cl, Lee J. Association of Vitamin D levels with outcome in patients with melanoma after adjustment for C-reactive protein. J Clinical Oncol. 2016;34(19):1741-7.

\section{Submit your next manuscript to BioMed Central and we will help you at every step:}

- We accept pre-submission inquiries

- Our selector tool helps you to find the most relevant journal

- We provide round the clock customer support

- Convenient online submission

- Thorough peer review

- Inclusion in PubMed and all major indexing services

- Maximum visibility for your research

Submit your manuscript at www.biomedcentral.com/submit 